\title{
Regularities of Magnetocaloric Effect and Determination of Some Thermodynamic Parameters for (Octaethylporphynato)chloro- manganese(III)
}

\author{
Victor V. Korolev, ${ }^{a}$ Marija E. Klyueva, ${ }^{b}$ Igorj M. Arefyev, $^{\text {a Anna G. Ramazanova, }}{ }^{a}$ \\ Tatyana N. Lomova, ${ }^{a} @$ and Anatolij G. Zakharov ${ }^{\mathrm{a}}$ \\ ${ }^{a}$ Institute of the Solution Chemistry of Russian Academy of Sciences, Ivanovo, 153045, Russia \\ ${ }^{\mathrm{b}}$ Ivanovo State University of Chemistry and Technology, Ivanovo, 153000, Ivanovo, Russia \\ ${ }^{\circledR}$ Corresponding authorE-mail: tnl@isc-ras.ru
}

\begin{abstract}
Magnetocaloric effect (MCE) and specific heat capacity of $(2,3,7,8,12,13,17,18$-octaethylporphynato)chloromanganese(III) (6\% suspension in water) were measured by microcalorimetric method in the dependence of temperature (298-353 K) and magnetic field (0-0.7 T). It is found that the MCE has positive value which increases with growth of the magnetic field induction at all the temperatures and decreases with the temperature growth at all the magnetic fields. Breaking the magnetic field leads to adiabatic demagnetizing. The dependence of specific heat capacity of the studied system from the value of magnetic field induction has maximum at 0.2-0.3 T at all the temperatures. When the induction value is higher than $0.6 \mathrm{~T}$ the specific heat capacity value is less than that in zero field. The temperature growth leads to the increase of the specific heat capacity in all the magnetic fields. On the base of the experimental data on the magnetocaloric effect and specific heat capacity of the manganese complex the dependencies of enthalpy, of the magnetic components of molar heat capacity and entropy from the temperature and magnetic induction are calculated.
\end{abstract}

\section{Introduction}

The high spin state of the central manganese atom in complexes with cyclic tetrapyrrole ligands is reliably confirmed by measurements of the magnetic susceptibility for their solid samples or solutions. Effective magnetic moment $\left(\mu_{\text {efff }}\right)$ value is equal $5.9 \div 6.6 \mu_{\mathrm{B}}$ for porphyrin complexes of manganese(II) and $4.8 \div 5.0 \mu_{\mathrm{B}}$ for the complexes of manganese(III) ${ }^{[1,2]}$ - the most stable form in the aromatic macrocyclic complexes.

Paramagnetic properties are used when studying and elaborating the building blocks of molecular magnets on the base of manganeseporphyrins and their tetraazaanalogs phthalocyanines. ${ }^{[3,4]}$ Complex macrocyclic core in these materials is a cation in the composition of a charge transfer complex or a free radical with unpaired electron on the macrocycle. Coordination polymers on the basis of tetrameso-substituted manganese(III) porphyrin tetracyanoethenide $^{[5,6]}\left[\mathrm{Mn}^{\mathrm{III}} \mathrm{P}\right]^{+}[\mathrm{TCNE}]^{-}$and oxidized diphthalocyanine $\mathrm{InPc}_{2}{ }^{\cdot[7]}$ are the examples. Measurements of magnetic susceptibility demonstrate typical Curie - Weiss behaviour for polycrystals of these compounds. The magnetocaloric effect (MCE) is well known in magnetochemistry, and in the present work we have studied for the first time its appearance for the aromatic macroheterocyclic complexes of manganese. The magnetic field dependences of the thermodynamic parameters of solid particles, namely the specific heat capacity, enthalpy changing and changing the magnetic component of the molar heat capacity and entropy were determined. Well characterized $\quad(2,3,7,8,12,13,17,18$-octaethylporphynato)chloromanganese(III) (Cl)MnOEP was chosen as an object.

\section{Experimental}

The measurements of MCE and specific heat capacity of water suspension of the manganese complex were made with the use of automatic microcalorimeter with isothermal cover. ${ }^{[8]}$ The microcalorimetric cell with the isothermal cover was placed into the interpoles clearance of the electromagnet $(60 \mathrm{~mm})$ (Figure 1). It allowed measurements of MCE and specific heat capacity values in magnetic fields from 0 to $0.7 \mathrm{~T}$ at temperatures $283 \div 315$ $\mathrm{K}$. Temperature changing was registered by the high accuracy voltmeter connected with a computer. Specially elaborated program allowed the online presentation of the temperature resistance in dependence of time and storage of the data to file for further analysis.

Heat capacity values were calculated according to equation (1):

$$
C_{\mathrm{p}}=\frac{Q_{\mathrm{C}}}{\Delta T}-\mathrm{W}
$$

where $Q_{\mathrm{C}}$ - quantity of heat (in $\mathrm{J}$ ) introduced into the calorimetric vessel by calibrated heater; $\Delta T$ - the change of the temperature in the calorimeter per joule of the introduced heat with account for heat exchange in initial and final periods; $W$ - the calorimeter constant ${ }^{[9]}$.

Sensitivity of the apparatus was $2 \cdot 10^{-5}{ }^{\circ} \mathrm{C}$, errors in the measurements of MCE and of the specific heat capacity were \pm $0.1 \%$ and $\pm 1 \%$, respectively.

$(2,3,7,8,12,13,17,18$-octaethylporphynato $)$ chloromanganese(III) (Cl)MnOEP was synthesized by Adler method ${ }^{[10]}$ and purified by chromatography on $\mathrm{Al}_{2} \mathrm{O}_{3} . v_{\max }(\mathrm{KBr}) / \mathrm{cm}^{-1} 728,749,841,962$, 989, 1019, 1055, 1062, 1112, 1148, 1272, 1315, 1373, 1451, 1464, 1480, 1604, 1632, 2931, 2872, 2966. $\lambda_{\max }\left(\mathrm{CHCl}_{3}\right)(\lg \varepsilon) / \mathrm{nm} 357$ (4.87), 428 (4.17), 473 (4.68), 559 (4.01), 590 (3.76), 687(3.22), 787(3.45). Water suspension of the complex was prepared by mixing of its weighted amount with bidistillate. Mass part of the solid sample was equal $6 \%$. 


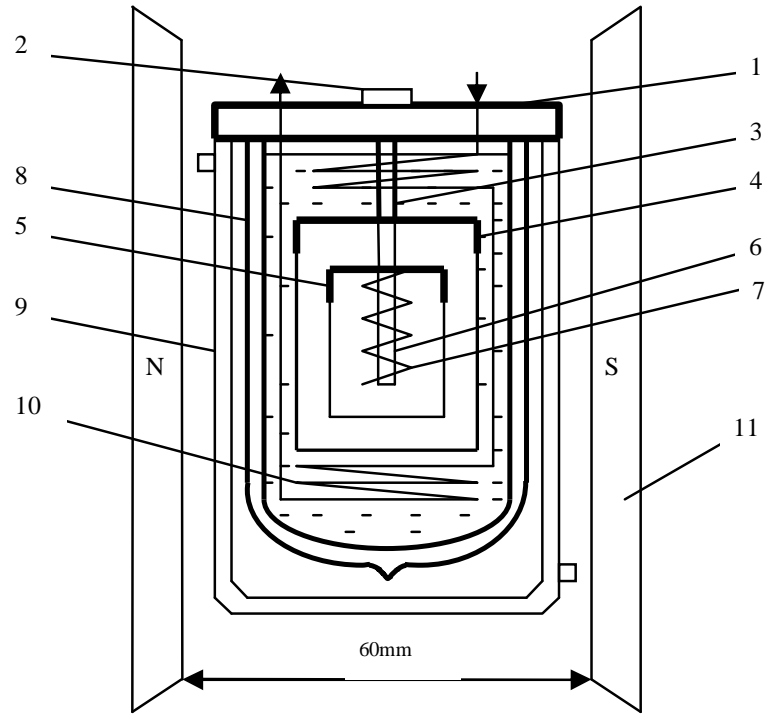

Figure 1. A schematic representation of the calorimetric cell: 1 - the cover with a plug connector; 2 - plug connector; 3 - bushing; 4 - cover of the outer cylinder of the isothermal shell; 5 -cover of the calorimetric vessel; 6 - casing with the thermistor; 7 - casing with the calibration heater; 8 - Dewar vessel; 9 - temperaturecontrolled glass jacket; 10 - heat exchanger; 11 - poles of electromagnet.

\section{Results and Discussions}

Previous systematic study of spectral characteristics of meso-tetraphenylporphyrin complexes of transition metals in connection with the structure and stability of the coordination spheres showed specificity of the manganese complexes in metalloporphyrin series ${ }^{[11]}$. Ratio of ionization potentials $\mathrm{Mn}^{3+} / \mathrm{Mn}^{2+}$ equal $33.69 / 15.64$ provides stability of the isolated $\mathrm{Mn}^{2+}$ cation. In complexes with tetrapyrrole macrocyclic ligands - porphyrins a formal charge of manganese cation may be equal $+2,+3,+4$ or +5 . Reaction of manganese(II) salts with porphyrins $\left(\mathrm{H}_{2} \mathrm{P}\right)$ in solution leads to formation of highly stable acidoporphyrin complexes of manganese(III). For example, redox potential of $\mathrm{Mn}^{\mathrm{III}} \mathrm{TPP} / \mathrm{Mn}^{\mathrm{II}} \mathrm{TPP}$ (TPP - meso-tetraphenylporphyrin dianion) pair is equal $-0.22 \quad \mathrm{~V}^{[12]}$ Stabilization of manganese oxidation state +3 is achieved due to action of the cyclic $\pi$-system of the organic ligand increasing the thermodynamic stability of the coordination bonds and the kinetic effects of the central metal screening as well.

As it is established by physical-chemical methods an absolute majority of manganese porphyrins are high spin compounds. This fully applies to manganese(III) complexes with $3 d^{4}$ electron configuration. Presence in $\mathrm{Mn}^{\text {III }}$ of halffilled $d_{\pi}$ and antibonding low energy $d_{\mathrm{x} 2 \text {-y2 }}$ orbitals promotes formation of $\pi$-bonds in the molecule in stationary and exited states along with coordination $\sigma$-bonds. Specificity of the electronic structure of manganese porphyrins is reflected in particular in revealing the magnetocaloric effect of $(\mathrm{Cl}) \mathrm{MnOEP}$ suspension in water.

Magnetocaloric effect takes place when magnetic field is applied to a substance having magnetic properties (magnetics, paramagnetic salts, rare-earth elements alloys). As a result of the action the heat is developed or absorbed due to the changing of the substance magnetic state and therefore its inner energy. In conditions of adiabatic heat isolation the temperature increase or decrease can be registered.

Heat capacity measurements are very important in the study of molecular magnetics, since the analysis of the temperature dependencies of the heat capacity in magnetic fields provides useful information about magnetic regulation of magneto active substances. It should be noted that MCE and heat capacity of macroheterocyclic complexes in magnetic fields were not studied earlier.

Figures 2-5 show experimental results of study of MCE and heat capacity of the $6 \%$ water suspension of the manganese complex. The observed MCE values are positive. They increase non-linearly with growth of the magnetic field induction and decrease with growth of the temperature in the studied ranges of magnetic induction and temperature (Figure 2,3). The slopes of polytherms rise when the field increases.

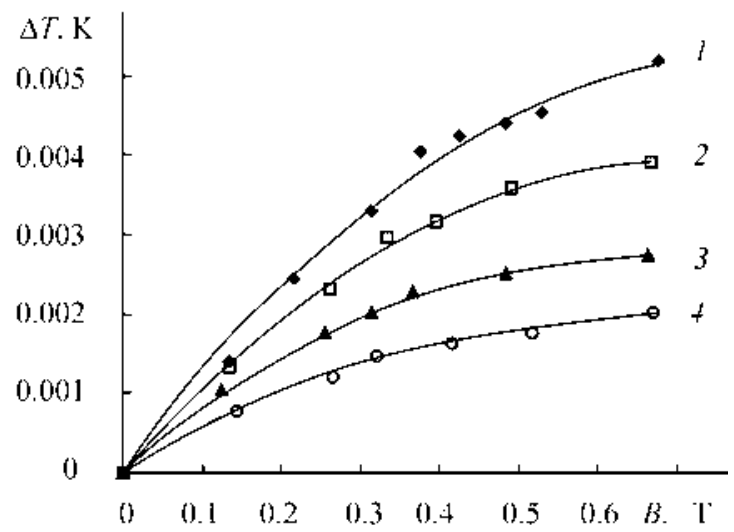

Figure 2. MCE of $6 \%$ water suspension of $(\mathrm{Cl}) \mathrm{MnOEP}$ as a function of applied magnetic field at different temperatures. T, K: 1 - 298, 2 - 313, 3-328, 4-343.

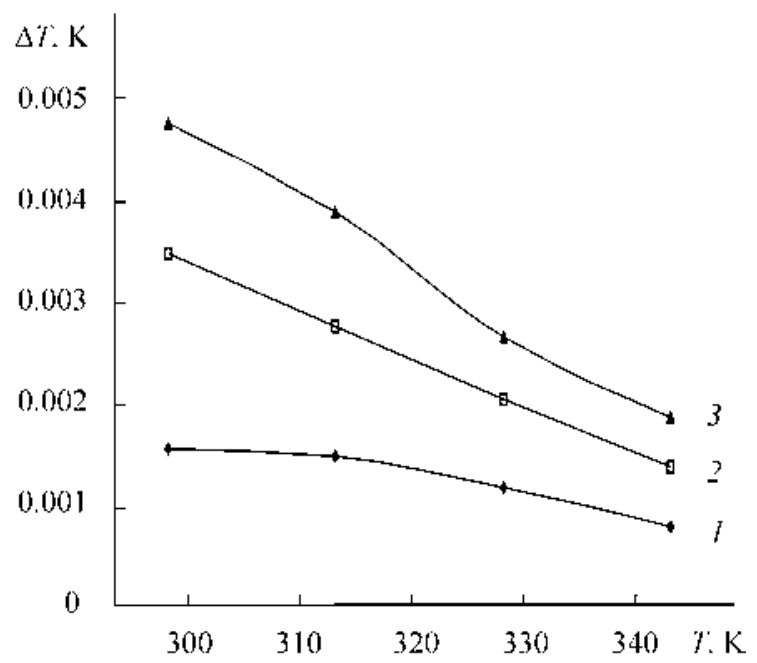

Figure 3. MCE of $6 \%$ water suspension of $(\mathrm{Cl}) \mathrm{MnOEP}$ as a function of temperature in various applied magnetic fields. $B, \mathrm{~T}: 1-0.15,2-0.32,3-0.6$.

Values of specific heat capacity of the manganese complex particles in magnetic field significantly differ from the heat capacity value in zero field (Figure 3). Dependencies of specific heat capacity from magnetic field 


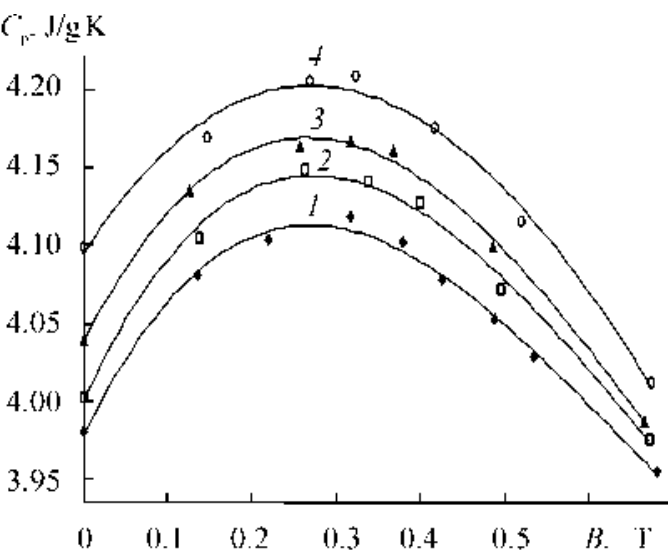

Figure 4. Specific heat capacity of $(\mathrm{Cl}) \mathrm{MnOEP}$ particles as a function of applied magnetic field. T, K: $1-298,2-313,3-328$, $4-343$.

induction are of the extreme character and have maxima at $0.25 \mathrm{~T}$. Heat capacity values at induction higher than 0.5 $0.6 \mathrm{~T}$ become less than in zero field. Tendency of the heat capacity values to decrease in the fields higher than $0.5 \mathrm{~T}$ evidences about changing of the complex magnetic structure. The presence of the heat capacity maximum is connected with magnetic structure of the particles. Earlier we have observed similar maxima for high disperse particles with ferrimagnetic and ferromagnetic structures. ${ }^{[13-}$ ${ }^{15]}$ It is difficult to give an interpretation of the maximum at the present stage of the study.

Specific heat capacity increases with the temperature growth at all values of the magnetic field (Figure 5).

With the use of the calorimetric equipment described above change of the enthalpy for the manganese complex particles in water suspension at applied magnetic field was determined by comparison of the temperature dependence of MCE and heat of the calibrated heater; heat exchange error in the calorimetric experiment was taken into account. Dependencies of the enthalpy from magnetic induction and temperature are analogous to MCE dependencies and are not shown here.

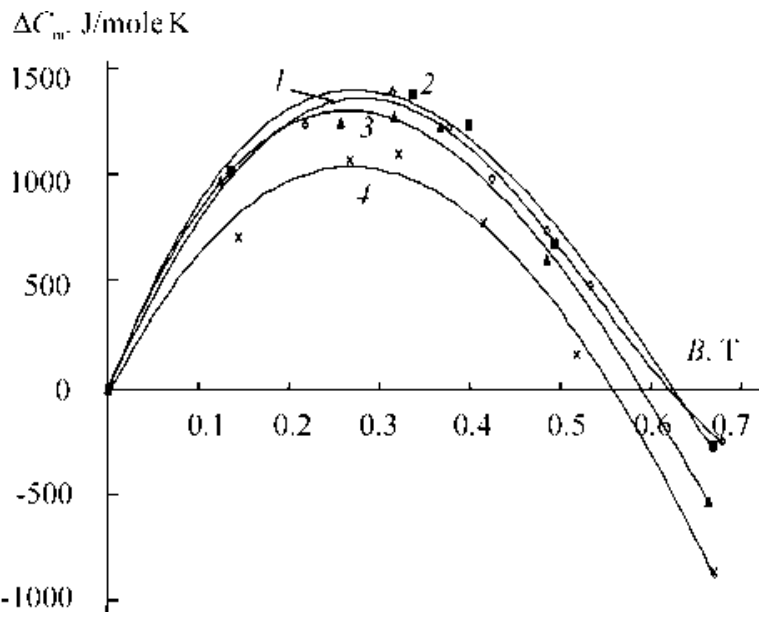

Figure 6. Changes of the magnetic part of the molar heat capacity for $(\mathrm{Cl}) \mathrm{MnOEP}$ particles as a function of applied magnetic field. $T, \mathrm{~K}: 1-298,2-313,3-328,4-343$.

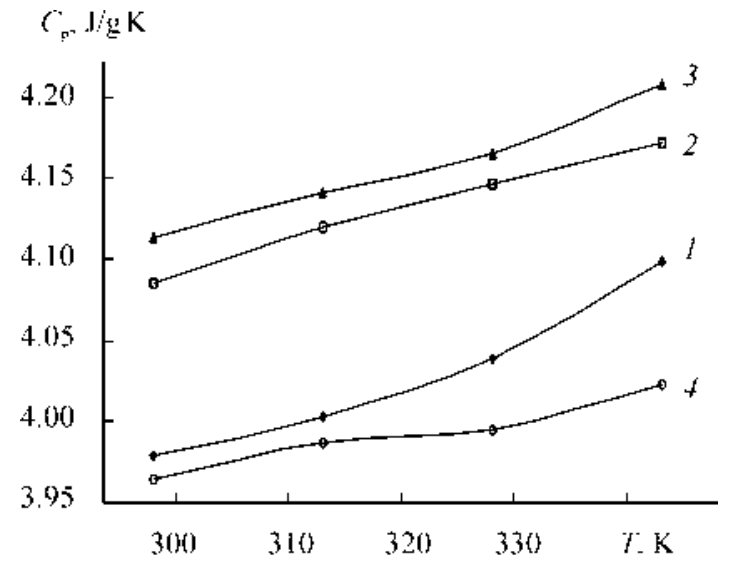

Figure 5. Specific heat of $(\mathrm{Cl}) \mathrm{MnOEP}$ particles as a function of temperature in various applied magnetic fields. $B, \mathrm{~T}: 1-0,2-$ $0.15,3-0.32,4-0.65$.

Only heat capacity of magnetic phase of water suspension, i.e. the manganese complex particle, undergoes changes at applied magnetic field. By subtraction of the heat capacity value in zero field from the value of specific heat capacity in current field the change of magnetic part of specific or molar heat capacity, in respect to 1 mole of the complex at constant induction and temperature, i.e. $\Delta C_{\mathrm{m}}(H, T)$, may be obtained. Induction and temperature dependencies of $\Delta C_{\mathrm{m}}(H, T)$ are shown in Figures 6 and 7. Their values are necessary for calculation of the dependencies of the magnetic part of entropy $\Delta S_{\mathrm{m}}(H, T)$ at applied magnetic field. The $\Delta S_{\mathrm{m}}(H, T)$ values were found according to thermodynamic equation (2): ${ }^{[16]}$

$$
\Delta S_{\mathrm{m}}(H, T)=\Delta T \Delta C_{\mathrm{m}}(H, T) / T
$$

where $\Delta T$ - magnetocaloric effect.

Dependencies of the obtained $\Delta S_{\mathrm{m}}(H, T)$ values from the applied magnetic field and temperature are shown in Figures 8 and 9.

Isotherms of the $\Delta S_{\mathrm{m}}(H, T)$ dependence from magnetic field induction have an extreme character with the maxima

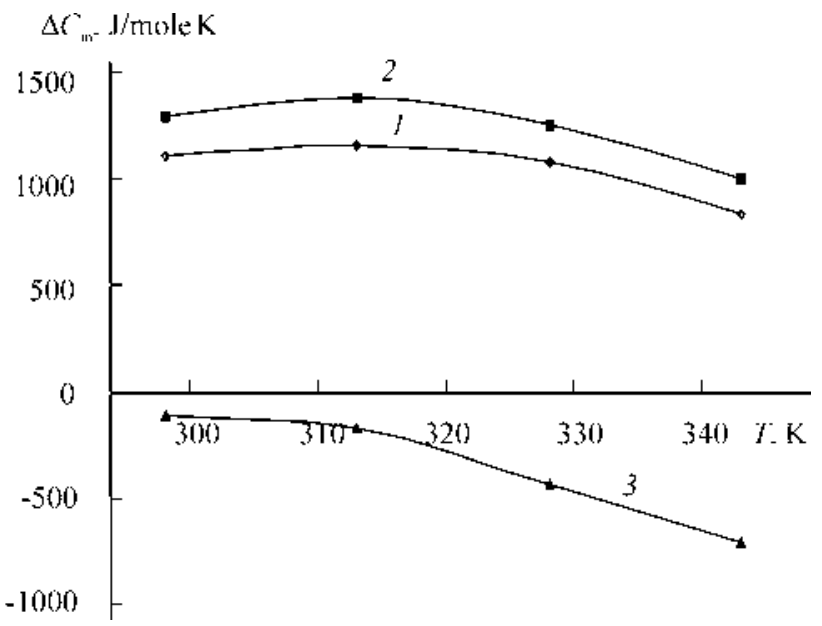

Figure 7. Changes of the magnetic part of the molar heat capacity for $(\mathrm{Cl}) \mathrm{MnOEP}$ particles as a function of temperature in various applied magnetic fields. $B, \mathrm{~T}: 1-0.15,2-0.32,3-0.65$. 
at induction equal $0.3 \div 0.4 \mathrm{~T}$ (Figure 8 ). The maximum height decreases when the temperature increase. The $\Delta S_{\mathrm{m}}(\mathrm{H}, \mathrm{T})$ values decrease with the temperature growth and become negative when induction is equal 0.65 $\mathrm{T}$ (Figure 9).

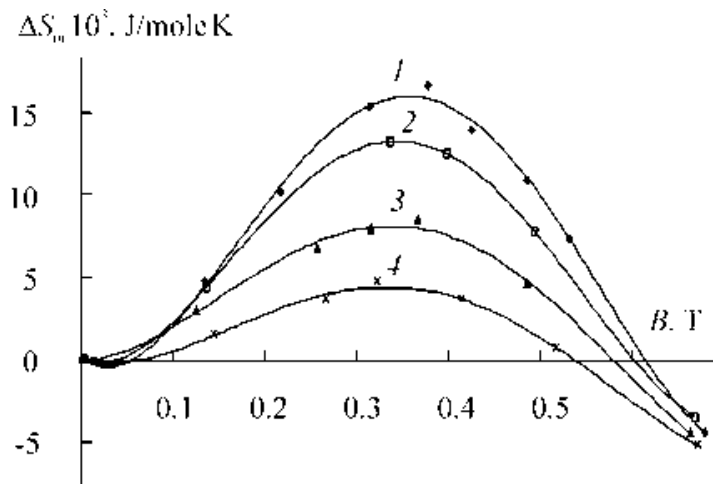

Figure 8. Changes of the magnetic part of molar entropy for (Cl)MnOEP particles as a function of applied magnetic field. $T, \mathrm{~K}$ : $1-298,2-313,3-328,4-343$.

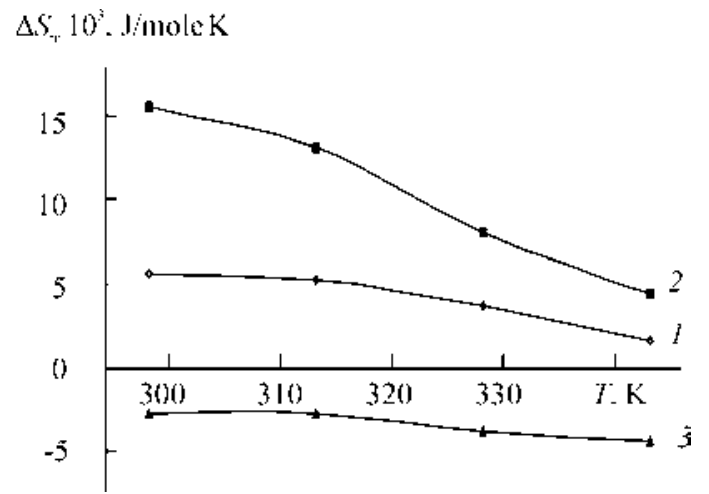

Figure 9. Changes of the magnetic part of the molar heat entropy for $(\mathrm{Cl}) \mathrm{MnOEP}$ particles as a function of temperature in various applied magnetic fields. $B, \mathrm{~T}: 1-0.15,2-0.32,3-0.65$.

\section{Conclusions}

It is found that $(2,3,7,8,12,13,17,18$-octaethylporphynato)chloromanganese(III) in aqua suspension reveals noticeable magnitocaloric effect which decreases with temperature increase.

For the first time specific heat capacity of solid particles of manganese porphyrin complex in magnetic field and its dependence from magnetic stream density are determined. Dependence of specific heat from applied magnetic field shows a maximum in medium fields.

Change of the magnetic part of the molar entropy for the manganese complex particles $\Delta S_{\mathrm{M}}(H, T)$ as a function of applied magnetic field has maximum at $0.3 \div 0.4 \mathrm{~T}$, the maximum height decreasing with temperature increase.

Thus, the method used allowed to obtain the thermodynamic characteristics of macroheterocyclic complex for the first time. For the further developing magnetochemistry of macroheterocyclic complexes it is necessary to study a series of the compounds having related structure.

Acknowledgements. The work is supported by Russian Foundation for Fundamental Research (grant 06-03-96343) and by the program N.8 of the Presidium of RAS "Elaborating of methods of synthesis of chemical substances and creating new materials".

\section{References}

1. Boucher L.J. Coord. Chem. Rev. 1972, 7, 289-329.

2. Walker F.A. Proton NMR and EPR Spectroscopy of Paramagnetic Metalloporphyrins, in The Porphyrin Handbook (Kadish K.M., Smith K.M., Guilard R., Eds.) Vol. 5. Amsterdam: Academic Press, 2000, 81-184.

3. Miller J.S., Calabrese J.C., McLean R.S., Epstein A.J. Adv. Mater. 1992, 4, 498.

4. Miller J.S., Vazquez C., Calabrese J.C., McLean R.S., Epstein A.J. Adv. Mater. 1994, 6, 217.

5. Böhm A., Vazquez C., McLean R.S., Calabrese J.C., Kalm S.E., Manson J.L., Epstein A.J., Miller J.S. Inorg. Chem. 1996, 35, 3083-3088.

6. Brandon E.J., Arif A.M., Burkhart B.M., Miller J.S. Inorg. Chem. 1998, 37, 2793-2798.

7. Janczak J., Kubiak R., Jezierski A. Inorg. Chem. 1995, 34, 3505-3508.

8. Korolev V.V., Romanov A.S., Arefyev I.M. Russ. J. Phys. Chem. A, Focus on Chem. 2006, 80, 310-312.

9. Perelygin I.S., Kimtis L.L., Tchizhik V.I. Experimental'niye metodi v khimii rastvorov: spektroskopiya I kalorimetriya [Experimental methods in solution chemistry: spectroscopy and calorimetry] Moskva, Nauka, 1995. 380

10. Adler A.D., Longo F.R., Kampus F., Kim J. J. Inorg. Nucl. Chem. 1970, 32, 2443-2445.

11. Lomova T.N., Berezin B.D. Russ. J. Coord. Chem. 2001, 27 , 85-104.

12. Mairanovsky V.G. Electrokhimiya porfirinov, in Porfirini: spektroskopiya, elektrokhimiya, primeneniye [Porphyrins: spectroscopy, electrochemistry, application] (Enikolopiyan, Ed.) Moskva, Nauka, 1987. 127.

13. Korolev V.V., Arefyev I.M., Ramazanova A.G. Russ. J. Phys. Chem. A, Focus on Chem. 2007, 81, 949-951.

14. Korolev V.V., Arefyev I.M., Ramazanova A.G. Russ. J. Phys. Chem. A, Focus on Chem. 2007, 81, 1677-1680.

15. Korolev V.V., Arefyev I.M., Ramazanova A.G. Rossiiskiye nanotechnologii 2007, 2, 59-62.

16. Vonsovsky S.V. Magnetizm [Magnetism] Moskva,: Nauka, 1971. 1032 